\title{
Error of displacement measurements using digital volume correlation and high resolution peripheral quantitative computed tomography
}

\author{
Dylan Zaluski ${ }^{1}$, Saija Kontulainen ${ }^{2}$, James (J.D.) Johnston ${ }^{1}$ \\ ${ }^{1}$ Department of Mechanical Engineering, University of Saskatchewan, Saskatoon, Canada \\ ${ }^{2}$ College of Kinesiology, University of Saskatchewan, Saskatoon, Canada
}

\begin{abstract}
Background: Digital volume correlation (DVC) is a 3D image processing technique for non-invasive assessment of internal deformation of structures in unloaded and loaded (deformed) states. One application of DVC is experimental validation of displacement predictions from subject-specific finite element (FE) models of bone. FE models can help clinicians and researchers better understand musculoskeletal diseases which affect bone mechanics (e.g., osteoporosis, osteoarthritis); however, FE models must be validated by experimental testing. Micro-CT is typically used for DVC due to its low noise and high resolution but the scanner is limited to small bone samples. High-resolution peripheral quantitative CT (HR-pQCT) can scan larger volumes, making it suitable for studying long bones affected by musculoskeletal disease (e.g., tibia, femur). The aim of this study was to estimate errors associated with DVC measures of displacement using HR-pQCT scans.

Methods: Five cadaveric proximal tibiae were scanned twice using HR-pQCT (integration time 300ms, isotropic voxel size $41 \mu \mathrm{m}$ ) with no external load. Due to limitations in detector size, each specimen scan was acquired as a series of 8 blocks of 220 slices each, with a 20 -slice overlap between each consecutive block. After reconstruction and ring artifact correction of all blocks, the full 8-block scan volumes were obtained by rigidly registering the overlapping blocks and linearly blending the common regions using a custom program in MATLAB. A variety of noise filters (median, bilateral, total variation diminishing and non-local means) were tested to identify which method resulted in the lowest displacement measurement error. Displacement fields for the zero-strain scans were obtained using a commercial DVC software (DaVis 10.01, LaVision Inc.). Measurement error of each displacement component was estimated by the standard deviation (SD) of the displacement fields from DVC. The least significant change (LSC) was calculated as $2.77 \mathrm{x}$ SD and represents the minimum displacement which can be measured with $95 \%$ confidence.

Results: With or without noise filtering, the SD of the $x, y$ and $z$ displacement components were 4.0, 9.0 and $8.5 \mu \mathrm{m}$ respectively, corresponding to LSCs of 11.1, 25.0 and $23.5 \mu \mathrm{m}$. Displacement errors were highest at the transition region between scan blocks due to registration inaccuracy.

Conclusion: Using HR-pQCT, internal bone displacements could be measured with sub-voxel accuracy (LSC of $25 \mu \mathrm{m}<41 \mu \mathrm{m}$ voxel size) for large scan volumes such as long bones. Advanced noise filtering techniques provided no improvement in results due to registration errors associated with the stitching process.
\end{abstract}

